\title{
A RELAXATION RESULT FOR ENERGIES DEFINED ON PAIRS SET-FUNCTION AND APPLICATIONS
}

\author{
Andrea Braides ${ }^{1}$, Antonin Chambolle $^{2}$ and Margherita Solci ${ }^{3}$
}

\begin{abstract}
We consider, in an open subset $\Omega$ of $\mathbb{R}^{N}$, energies depending on the perimeter of a subset $E \subset \Omega$ (or some equivalent surface integral) and on a function $u$ which is defined only on $\Omega \backslash E$. We compute the lower semicontinuous envelope of such energies. This relaxation has to take into account the fact that in the limit, the "holes" $E$ may collapse into a discontinuity of $u$, whose surface will be counted twice in the relaxed energy. We discuss some situations where such energies appear, and give, as an application, a new proof of convergence for an extension of Ambrosio-Tortorelli's approximation to the Mumford-Shah functional.
\end{abstract}

Mathematics Subject Classification. 49J45, 49Q20, 49Q10.

Received December 12, 2005. Revised July 17, 2006.

Published online July 20, 2007.

\section{INTRODUCTION}

In this paper we consider energies defined on pairs function/open subset of $\mathbb{R}^{n}$, of the form

$$
F(u, E)=\int_{\Omega \backslash E} f(\nabla u) \mathrm{d} x+\int_{\partial E} \varphi(\nu) \mathrm{d} \mathcal{H}^{n-1},
$$

with interacting bulk and surface energies. Here $E$ is thought to be smooth enough (e.g., with Lipschitz boundary) so that $\partial E$ coincides $\mathcal{H}^{n-1}$-a.e. with the essential boundary of $E$; i.e., with the interface between the 'interior' and 'exterior' of $E$, and $u \in W^{1, p}\left(\Omega ; \mathbb{R}^{m}\right)$. Energies of this type arise in physical problems for example when dealing with small drops or thin films, when bulk and surface energies can be thought to be of the same order (see, e.g., [7] for a variational problem set in this framework).

Functionals as $F$ appear also in some disguised form in many problems related to variational models in Image Segmentation, such as that by Mumford and Shah [22,23]. A particularly successful approach to deal with such problems has proven to be the application of the direct methods of the Calculus of Variations in the framework of the special functions of bounded variation to obtain existence and regularity results. In order to apply these existence results to Image Segmentation problems a further step is necessary, of approximating free-discontinuity energies, containing competing bulk and surface integrals, by energies to which numerical

\footnotetext{
Keywords and phrases. Relaxation, free discontinuity problems, $\Gamma$-convergence.

1 Dip. di Matematica, Università di Roma "Tor Vergata", via della Ricerca Scientifica, 00133 Roma, Italy.

2 CMAP, École Polytechnique, CNRS, 91128 Palaiseau, France; antonin.chambolle@polytechnique.fr

3 DAP, Università di Sassari, Palazzo Pou Salit, 07041 Alghero, Italy.
} 
methods can be more easily applied. This has been done in many different ways, using elliptic energies with an additional variable, finite-difference energies, non-local integrals, etc. (see [11]). A commom pattern can be traced in all those approximations, that start from an ansatz on the desired form of an 'approximate solution'. While it is easily seen, by construction of the approximating energies, that the candidate approximate solutions give the desired limit energy, more elaborate arguments are usually necessary to prove that the envisaged form of the solutions is 'optimal'; i.e., that substantially lower energies cannot be achieved using a different type of pattern. To prove this fact (in the language of $\Gamma$-convergence we would call this the 'liminf inequality') a crucial point is, given an arbitrary sequence of minimizers, to distinguish sets in which the approximating energies computed on these functions behave as 'bulk energies', and complementary sets which we may regard as 'blurred' discontinuity sets (typically these are sets where 'gradients are high'). This point can be rephrased as comparing the candidate approximating energies with an energy as $F$ above defined on pairs function-set, whose form is in general dependent only on the 'target' free-discontinuity energy. In the case of the Mumford and Shah functional

$$
M S(u)=\int_{\Omega}|\nabla u|^{2} \mathrm{~d} x+\mathcal{H}^{n-1}(S(u) \cap \Omega)
$$

$(S(u)$ denotes the set of discontinuity points of $u$ ), in this process of proving the liminf inequality we often produce energies of the form

$$
\widetilde{F}(u, E)=a \int_{\Omega \backslash E}|\nabla u|^{2} \mathrm{~d} x+b \mathcal{H}^{n-1}(\Omega \cap \partial E),
$$

with $a$ and $b$ constants belonging to some sets that depend on the specific argument used. The 'liminf inequality' is then rephrased in terms of the lower semicontinuous envelope of $\widetilde{F}$, and a localization argument showing that we may separately optimize the choice of $a$ and $b$. This remark is essentially already contained in a paper by Bourdin and Chambolle [10], but therein it is not stated explicitly in terms of a relaxation result, and is obtained by applying more elaborated approximation results.

Note that at fixed $E$ the functional $F(\cdot, E)$ is weakly lower semicontinuous on $W^{1, p}$, provided some standard convexity and growth conditions on $f$ are required, and that, at fixed $u, F(u, \cdot)$ can be extended to a lower semicontinuous energy on sets with finite perimeter if $\varphi$ is a norm (see for instance [3]). On the contrary, the functional defined on pairs $(u, E)$ is not lower semicontinuous. Loosely speaking, if $\left(u_{j}, E_{j}\right)$ is a sequence with equi-bounded energy and converging to some $(u, E)$, the limit $u$ may be discontinuous on $\Omega \backslash E$, and its set of discontinuity points $S(u)$ may be the limit of a portion of $\partial E_{j}$. In this paper we compute the lowersemicontinuous envelope of functionals $F$ in a direct way, and characterize it in the whole class of pairs $(u, E)$, where $E$ is a set of finite perimeter and $u$ is such that $u\left(1-\chi_{E}\right)$ belongs to the space $G S B V\left(\Omega ; \mathbb{R}^{m}\right)$ of Ambrosio and De Giorgi's generalized special function of bounded variation (Th. 2). We show that it takes the form

$$
\bar{F}(u, E)=\int_{\Omega \backslash E} f(\nabla u) \mathrm{d} x+\int_{\partial^{*} E \cap \Omega} \varphi(\nu) \mathrm{d} \mathcal{H}^{n-1}+\int_{S(u) \cap \Omega \cap E_{0}} \widetilde{\varphi}(\nu) \mathrm{d} \mathcal{H}^{n-1},
$$

where $E_{0}$ denotes the points of density zero of $E$ and $\widetilde{\varphi}(\nu)=\varphi(\nu)+\varphi(-\nu)$. The result is proved by providing separately a lower bound and an upper bound. The lower bound is obtained by reducing to the one dimensional case through Ambrosio's slicing techniques. The crucial technical point is here to check that, loosely speaking, for almost all directions, the traces of one-dimensional sections of $E$ have density 0 on the jump set $S(u)$ precisely on the intersection of $E_{0}$ and $S(u)$ (Lem. 5). The upper bound is obtained by a direct construction if $S(u)$ is smooth enough, and by approximation in the general case. We show two ways to obtain such an approximation. The first one (Lem. 12) consists in applying a 'strong SBV approximation' result by Braides and Chiadò Piat [14] to a suitable modification of the function $u\left(1-\chi_{E}\right)$, and then construct optimal pair from this sequence of approximating functions. The second one (Rem. 13) uses a mollification argument for approximating the set $E$ first, and then the coarea formula to select approximating sets, on which then to obtain an approximation of the target $u$. 
As applications of this result, we first give an approximation of the Mumford-Shah energy by a sequence of functions defined on pairs set-functions, by noting that $\bar{F}(u, E)$ reduces to a functional on $G S B V(\Omega)$ when $E=\emptyset$, so that $E_{0}=\Omega$. Subsequently, we give a different proof of Ambrosio-Tortorelli's elliptic approximation result [4]. At the same time we provide a generalization by replacing their one-well potential by perturbed double-well potentials, which give a different smoother form of the optimal profile of solutions. This result formalizes a method that has already been used by Braides and March [15] to obtain minimizing sequences bounded in $H^{2}$ for problems in which a term penalizing the curvature of the discontinuity set is added. Finally, we outline applications to the study of crystalline films on a substrate and to water waves. As an interesting additional object for perspective work, we mention the interaction with boundary conditions and microgeometry, that would lead to interesting problems of homogenization, as shown by Alberti and De Simone [1] already in the case when no bulk term is present (see also an earlier paper by Bouchitté and Seppecher [8]). As a final bibliographical information, the results in this paper concerning the Ambrosio-Tortorelli approximation previously circulated in the form of the manuscript [16].

\section{The Relaxation RESUlt}

Let $f: \mathbb{R}^{m \times n} \rightarrow \mathbb{R}$ be a quasiconvex function satisfying the growth condition

$$
c_{1}\left(|\xi|^{p}-1\right) \leq f(\xi) \leq c_{2}\left(1+|\xi|^{p}\right)
$$

for some positive constants $c_{1}$ and $c_{2}$, and $p>1$, and let $\varphi: \mathbb{R}^{n} \rightarrow[0,+\infty)$ be a convex and positively homogeneous function of degree one, with $\varphi(z)>0$ if $z \neq 0$. For every $u \in L^{1}(\Omega)$ and $E$ measurable subset of $\Omega$, we define

$$
F(u, E)= \begin{cases}\int_{\Omega \backslash E} f(\nabla u) \mathrm{d} x+\int_{\Omega \cap \partial E} \varphi\left(\nu_{E}\right) \mathrm{d} \mathcal{H}^{n-1} & \text { if } u \in W^{1, p}\left(\Omega ; \mathbb{R}^{m}\right) \text { and } \partial E \text { Lipschitz } \\ +\infty & \text { otherwise, }\end{cases}
$$

where $\nu_{E}$ denotes the interior normal to $E$.

We will prove the following relaxation theorem, in whose statement we adopt standard notation for generalized functions of bounded variations (see [5]); in particular, $S(u)$ and $\nu_{u}$ denote the set of essential discontinuity points of $u$ and its measure-theoretical normal, respectively. We say that $E_{j} \rightarrow E$ if $\chi_{E_{j}} \rightarrow \chi_{E}$ in $L^{1}(\Omega) ; M(\Omega)$ denotes the family of measurable subsets of $\Omega$ and $G S B V\left(\Omega ; \mathbb{R}^{m}\right)$ the space of $\mathbb{R}^{m}$-valued generalized special functions of bounded variation on $\Omega$.

Theorem 1. The lower-semicontinuous envelope of the functional $F$, with respect to the $L^{1}\left(\Omega ; \mathbb{R}^{m}\right) \times L^{1}\left(\Omega ; \mathbb{R}^{m}\right)$ topology, is the functional $\bar{F}: L^{1}\left(\Omega ; \mathbb{R}^{m}\right) \times \mathcal{M}(\Omega) \rightarrow[0,+\infty]$ defined as:

$$
\bar{F}(u, E)=\left\{\begin{array}{c}
\int_{\Omega \backslash E} f(\nabla u) \mathrm{d} x+\int_{\Omega \cap \partial^{*} E} \varphi\left(\nu_{E}\right) \mathrm{d} \mathcal{H}^{n-1}+\int_{\Omega \cap S(u) \cap E_{0}}\left(\varphi\left(\nu_{u}\right)+\varphi\left(-\nu_{u}\right)\right) \mathrm{d} \mathcal{H}^{n-1} \\
\text { if } u \chi_{E_{0}} \in G S B V\left(\Omega ; \mathbb{R}^{m}\right) \\
+\infty \\
\text { otherwise, }
\end{array}\right.
$$

where $\partial^{*} E$ is the reduced boundary of $E$ and $E_{0}$ is the set of the points where $E$ has density 0.

Furthermore, if $0<|E| \leq|\Omega|$ then for every pair $(u, E)$ there exists a recovery sequence $\left(u_{j}, E_{j}\right)$ such that $\lim _{j} F\left(u_{j}, E_{j}\right)=\bar{F}(u, E)$ and $\left|E_{j}\right|=|E|$.

The proof of Theorem 1 will be given in Sections 3 and 4, by showing, respectively, a lower and an upper inequality. By the nature of the problem we follow a 'hybrid' approach to the liminf inequality. In fact, on one hand we may regard the functional $F$ as the restriction of a functional $G$ defined on $G S B V\left(\Omega ; \mathbb{R}^{m}\right)$ to functions $v=u \chi_{\Omega \backslash E}$. A simple lower-semicontinuity argument applied to this functional $G$ gives the optimal lower bound 
for the bulk term of $\bar{F}$ (Rem. 7). This argument is not optimal for the surface energies in $\bar{F}$, for which we follow a different path by using a slicing argument. Since the argument for this part is essentially scalar and does not involve the bulk energy density $f$, the proof will be given in detail in the case when $m=1, f(\nabla u)=a|\nabla u|^{2}$ and $\varphi(z)=b|z| / 2$ (so that $\varphi(\nu)+\varphi(-\nu)=b$ if $|\nu|=1$ ) not to overburden notation, while the extension of the proof to the general case is given at the end of each section.

\section{The LOWER Inequality}

For every $u \in L^{1}(\Omega)$ and $E$ measurable subset of $\Omega$, we define

$$
F(u, E)= \begin{cases}a \int_{\Omega \backslash E}|\nabla u|^{2} \mathrm{~d} x+\frac{b}{2} \mathcal{H}^{n-1}(\Omega \cap \partial E) & \text { if } u \in H^{1}(\Omega) \text { and } \partial E \text { Lipschitz } \\ +\infty & \text { otherwise }\end{cases}
$$

where $a, b$ are positive parameters. For this choice of $F$ Theorem 1 reads as follows.

Theorem 2. The lower-semicontinuous envelope of the functional $F$ with respect to the $L^{1}(\Omega) \times L^{1}(\Omega)$ topology, is the functional $\bar{F}: L^{1}(\Omega) \times \mathcal{M}(\Omega) \rightarrow[0,+\infty]$ defined as:

$$
\bar{F}(u, E)=\left\{\begin{array}{cc}
a \int_{\Omega \backslash E}|\nabla u|^{2} \mathrm{~d} x+\frac{b}{2} \mathcal{H}^{n-1}\left(\partial^{*} E \cap \Omega\right)+b \mathcal{H}^{n-1}\left(S(u) \cap \Omega \cap E_{0}\right) \\
\text { if } u \chi_{E_{0}} \in G S B V(\Omega) \\
+\infty & \text { otherwise, }
\end{array}\right.
$$

where $\partial^{*} E$ is the reduced boundary of $E$ and $E_{0}$ is the set of the points where $E$ has density 0.

In order to prove a lower bound for the relaxation of $F$ we will use the 'slicing' method (see [12], Chap. 15) that allows to reduce to the study of energies defined on one-dimensional sections. To this end we will need to define as customary the 'localized' versions of our energies as follows. For every $u \in L^{1}(\Omega), E$ measurable subset of $\Omega$, and for every $A$ open subset of $\Omega$, we define

$$
F(u, E ; A)= \begin{cases}a \int_{A \backslash E}|\nabla u|^{2} \mathrm{~d} x+\frac{b}{2} \mathcal{H}^{n-1}(\partial E \cap A) & \text { if } u \in H^{1}(\Omega) \text { and } \partial E \text { Lipschitz } \\ +\infty & \text { otherwise. }\end{cases}
$$

Similarly, we define $\bar{F}(u, E ; A)$.

Setting, for every $u \in L^{1}(\Omega), E \in \mathcal{M}(\Omega)$ and $A$ open subset of $\Omega$ :

$$
F^{\prime}(u, E ; A)=\inf \left\{\liminf _{j \rightarrow \infty} F\left(u_{j}, E_{j} ; A\right): u_{j} \rightarrow u, E_{j} \rightarrow E \text { in } L^{1}(\Omega)\right\},
$$

we show the following inequality:

$$
F^{\prime}(u, E ; A) \geq \bar{F}(u, E ; A) .
$$

This corresponds to proving the following

Proposition 3. Let $u \in L^{1}(\Omega), E \in \mathcal{M}(\Omega)$ and $A$ be an open subset of $\Omega$. For every sequence $\left\{\left(u_{j}, E_{j}\right)\right\}$ in $L^{1}(\Omega) \times \mathcal{M}(\Omega)$, such that $u_{j} \rightarrow u$ and $E_{j} \rightarrow E$ in $L^{1}(\Omega)$ :

$$
\liminf _{j \rightarrow+\infty} F\left(u_{j}, E_{j} ; A\right) \geq \bar{F}(u, E ; A) .
$$


Proof. We start by stating the result concerning the one-dimensional functionals.

\section{- The lower inequality in the 1-dimensional case}

Let $I \subset \mathbb{R}$ be an interval, $u \in L^{1}(I)$ and let $E$ be a measurable subset of $I$. Let $\left\{u_{j}\right\}$ be a sequence in $L^{1}(I)$, and let $\left\{E_{j}\right\}$ be a sequence of measurable subsets of $I$, such that

$$
u_{j} \rightarrow u \text { in } L^{1}(I) \text { and } \quad E_{j} \rightarrow E \quad \text { as } j \rightarrow+\infty
$$

We can assume $\sup _{j} F\left(u_{j}, E_{j} ; I\right) \leq c$; then, $u_{j} \in H^{1}(I)$, the number of the connected components of $E_{j}$ is uniformly bounded, and we can find a finite set $S=\left\{s_{1}, \ldots, s_{L}\right\} \subset E_{0}$, a finite set of intervals $\left\{\left[a_{1}, b_{1}\right], \ldots,\left[a_{M}, b_{M}\right]\right\}$ and a subsequence (not relabelled) such that

$$
\forall \eta>0 \quad \exists \bar{j} \in \mathbb{N} \quad \forall j \geq \bar{j}: E_{j} \subset \bigcup_{i=1}^{L}\left[s_{i}-\eta, s_{i}+\eta\right] \cup \bigcup_{i=1}^{M}\left[a_{i}-\eta, b_{i}+\eta\right]
$$

In particular, we can assume $E=\bigcup_{i=1}^{M}\left[a_{i}, b_{i}\right]$. Then, setting $\Lambda_{\eta}=\bigcup_{i=1}^{L}\left[s_{i}-\eta, s_{i}+\eta\right]$ and $E_{\eta}=\bigcup_{i=1}^{M}\left[a_{i}-\eta, b_{i}+\eta\right]$ :

$$
\int_{I \backslash\left(\Lambda_{\eta} \cup E_{\eta}\right)}\left|u_{j}^{\prime}\right|^{2} \mathrm{~d} x \leq c ;
$$

by the arbitrariness of $\eta>0$, it follows that $u \in H^{1}((I \backslash E) \backslash S)$, hence $u \in S B V(I \backslash E)$ and $S(u) \cap E_{0} \subset S$. This allows to conclude

$$
\liminf _{j \rightarrow+\infty} F\left(u_{j}, E_{j} ; I\right) \geq \bar{F}(u, E ; I)
$$

It is easy to check that for every $J$ open subset of $I$ :

$$
\liminf _{j \rightarrow+\infty} F\left(u_{j}, E_{j} ; J\right) \geq \bar{F}(u, E ; J) .
$$

\section{- The lower inequality in the $n$-dimensional case}

We recall some definitions and properties related to the slicing procedure (for a general overview of this method we refer e.g., to [13], Sect. 3.4 or [11], Sect. 4.1). For every $\xi \in S^{n-1}$ let $\Pi_{\xi}$ be the $(n-1)$-dimensional linear subspace of $\mathbb{R}^{n}$ orthogonal to $\xi$. If $B \subseteq \mathbb{R}^{n}$ then $B^{\xi}$ be the orthogonal projection of $B$ on $\Pi_{\xi}$. For every $y \in B^{\xi}$ set $B^{\xi y}=\{t \in \mathbb{R}: y+t \xi \in B\}$. If $f: B \rightarrow \mathbb{R}$ let $f^{\xi y}: B^{\xi, y} \rightarrow \mathbb{R}$ be defined by $f^{\xi, y}(t)=f(y+t \xi)$.

The following results hold [5]:

(i) Let $u \in G S B V(\Omega)$ and let $D^{k} u$ stand for any of $D^{a}, D^{j}$ or $D^{c}$ (the absolutely continuous, jump or Cantor part of the derivative). Then, for every $\xi \in S^{n-1}$ and for $\mathcal{H}^{n-1}$-a.e. $y \in \Omega^{\xi}$ we have $u^{\xi y} \in G S B V\left(\Omega^{\xi y}\right)$; moreover, denoting by $\left\langle D^{k} u, \xi\right\rangle$ the component of $D^{k} u$ along $\xi$, the following representation holds:

$$
\int_{B}\left\langle D^{k} u, \xi\right\rangle=\int_{B^{\xi}} D^{k} u^{\xi y}\left(B^{\xi y}\right) \mathrm{d} \mathcal{H}^{n-1}(y)
$$

(ii) Let $u \in L^{1}(\Omega)$; assume that $u^{\xi y} \in S B V\left(\Omega^{\xi y}\right)$ for every $\xi$ in a basis of $\mathbb{R}^{n}$ and for a.e. $y \in \Omega^{\xi}$, and that

$$
\int_{\Omega^{\xi}}\left|D u^{\xi y}\right|\left(\Omega^{\xi y}\right) \mathrm{d} \mathcal{H}^{n-1}(y)<+\infty
$$

Then $u \in S B V(\Omega)$. Before proceeding in the proof we recall a result concerning the supremum of a family of measures (see [12], Lem. 15.2). 
Proposition 4. Let $\Omega$ be an open subset of $\mathbb{R}^{n}$ and $\mu$ a finite, positive set function defined on the family of open subsets of $\Omega$. Let $\lambda$ be a positive Borel measure on $\Omega$, and $\left\{g_{i}\right\}_{i \in \mathbb{N}}$ a family of positive Borel functions on $\Omega$. Assume that $\mu(A) \geq \int_{A} g_{i} \mathrm{~d} \lambda$ for every $A$ and $i$, and that $\mu(A \cup B) \geq \mu(A)+\mu(B)$ whenever $A, B \subset \subset \Omega$ and $\bar{A} \cap \bar{B}=\emptyset$ (superadditivity). Then $\mu(A) \geq \int_{A}\left(\sup _{i} g_{i}\right) \mathrm{d} \lambda$ for every $A$.

Moreover, we will use the following property.

Lemma 5. Let $\Gamma \subset E_{0}$ be a $(n-1)$-rectifiable subset, $\xi \in S^{n-1}$ such that $\xi$ is not orthogonal to the normal $\nu_{\Gamma}$ to $\Gamma$ at any point of $\Gamma$; then, for almost every $y \in \Pi_{\xi}$, for every $t \in \Gamma^{\xi y}$, $E^{\xi y}$ has density 0 in $t$.

Proof of Lemma 5. Since $\Gamma$ is contained in a countable union of $C^{1}$ hypersurfaces, up to localization on one of those surfaces and a deformation argument, we can assume $\Gamma \subset \Pi_{\xi}$.

We set:

$$
\Gamma_{k}=\left\{y \in \Gamma: \Theta\left(0, E^{\xi y}\right) \geq \frac{1}{k}\right\}, \quad \Gamma^{+}=\bigcup_{k \in \mathbb{N}} \Gamma_{k},
$$

where $\Theta\left(0, E^{\xi y}\right)$ denotes the (one-dimensional) density of the set $E^{\xi y}$ in 0 . In the following $Q_{\rho}^{\xi}(x)$ denotes a cube with centre $x$, side $\rho$, and two parallel faces orthogonal to $\xi$.

Let us assume by contradiction, that $\mathcal{H}^{n-1}\left(\Gamma^{+}\right)>0$. Then, there exists $k$ such that $\mathcal{H}^{n-1}\left(\Gamma^{k}\right)>0$. Since $\Gamma_{k} \subset E_{0}$, Lebesgue's dominated convergence theorem gives

$$
\lim _{\varrho \rightarrow 0} \int_{\Gamma_{k}} \frac{\left|E \cap Q_{\varrho}^{\xi}(x)\right|}{\varrho^{n}} \mathrm{~d} \mathcal{H}^{n-1}(x)=0
$$

Then

$$
\begin{aligned}
0 & =\liminf _{\varrho \rightarrow 0} \frac{1}{\varrho^{n-1}} \int_{\Gamma_{k}} \int_{\Gamma_{k} \cap Q_{\varrho}^{\xi}(x)} \frac{\left|E^{\xi y} \cap(-\varrho / 2, \varrho / 2)\right|}{\varrho} \mathrm{d} \mathcal{H}^{n-1}(y) \mathrm{d} \mathcal{H}^{n-1}(x) \\
& =\liminf _{\varrho \rightarrow 0} \frac{1}{\varrho^{n-1}} \int_{\Gamma_{k}} \int_{\Gamma_{k} \cap Q_{\varrho}^{\xi}(y)} \frac{\left|E^{\xi y} \cap(-\varrho / 2, \varrho / 2)\right|}{\varrho} \mathrm{d} \mathcal{H}^{n-1}(x) \mathrm{d} \mathcal{H}^{n-1}(y) \\
& =\liminf _{\varrho \rightarrow 0} \int_{\Gamma_{k}}\left(\frac{1}{\varrho^{n-1}}\left|\Gamma_{k} \cap Q_{\varrho}^{\xi}(y)\right|\right) \frac{\left|E^{\xi y} \cap(-\varrho / 2, \varrho / 2)\right|}{\varrho} \mathrm{d} \mathcal{H}^{n-1}(y) \\
& \geq \int_{\Gamma_{k}} \liminf _{\varrho \rightarrow 0} \frac{\left|E^{\xi y} \cap(-\varrho / 2, \varrho / 2)\right|}{\varrho} \mathrm{d} \mathcal{H}^{n-1}(y) \geq \frac{\mathcal{H}^{n-1}\left(\Gamma_{k}\right)}{k}
\end{aligned}
$$

This gives the contradiction.

Now we apply the slicing method to complete the proof of Proposition 3, in a fashion similar to that in [9].

Let $\phi$ and $\bar{\phi}$ denote the one dimensional versions of the functionals $F$ and $\bar{F}$, respectively. For every $\xi \in S^{n-1}$ we define $F^{\xi}: L^{1}(\Omega) \times \mathcal{M}(\Omega) \times \mathcal{A}(\Omega) \rightarrow[0,+\infty]$ as

$$
F^{\xi}(v, B ; A)=\int_{\Pi_{\xi}} \phi\left(v^{\xi, y}, B^{\xi, y} ; A^{\xi, y}\right) \mathrm{d} \mathcal{H}^{n-1}(y) .
$$

Note that $F \geq F^{\xi}$ for every $\xi$.

An application of the Fatou Lemma and the one dimensional inequality (4) give

$$
F^{\prime}(u, E ; A) \geq \bar{F}^{\xi}(u, E ; A)
$$


where, for $v \in L^{1}(\Omega)$ and $B \in \mathcal{P}(\Omega)$ :

$$
\bar{F}^{\xi}(v, B ; A)=\int_{\Pi_{\xi}} \bar{\phi}\left(v^{\xi y}, B^{\xi y} ; A^{\xi y}\right) \mathrm{d} \mathcal{H}^{n-1}(y) .
$$

Thus, if $F^{\prime}(u, E ; A)$ is finite, it follows that $E^{\xi y}$ is a finite union of intervals which we can suppose closed, and that $u^{\xi y} \in S B V\left(A^{\xi y} \backslash E^{\xi y}\right)$ for a.a. $y$ in $\Pi_{\xi}$; moreover

$$
\int_{\Pi_{\xi}}\left(\int_{A^{\xi y} \backslash E^{\xi y}}\left|\left(u^{\xi y}\right)^{\prime}\right|^{2} \mathrm{~d} t+\mathcal{H}^{0}\left(\left(S\left(u^{\xi y}\right) \cap\left(E^{\xi y}\right)_{0} \cap A^{\xi y}\right)\right)\right) \mathrm{d} \mathcal{H}^{n-1}(y)<+\infty .
$$

Then, if in addition $u \in L^{\infty}(\Omega)$, we get

$$
\int_{\Pi_{\xi}}\left|D\left(u^{\xi y}\right)\right|\left(A^{\xi, y} \backslash E^{\xi y}\right) \mathrm{d} \mathcal{H}^{n-1}(y)<+\infty .
$$

Recalling (ii), we deduce that, assuming $u \in L^{\infty}(\Omega)$, if $F^{\prime}(u, E ; A)$ is finite, then $u \chi_{E_{0}} \in S B V(A)$. A truncation argument allows to conclude that $F^{\prime}(u, E ; A)$ is finite only if $u \chi_{E_{0}} \in G S B V(A)$; in order to conclude applying (i), we need to prove that $S\left(u^{\xi y}\right) \cap\left(E_{0}\right)^{\xi y} \subset\left(S\left(u^{\xi y}\right) \cap E^{\xi y}\right)_{0}$; this follows from Lemma 5 , with $\Gamma=S(u) \cap E_{0} \cap$ $\left\{\left\langle\xi, \nu_{u}\right\rangle \neq 0\right\}$ Now, recalling (i), we get:

$$
\bar{F}^{\xi}(u, E ; A)=a \int_{A}|\langle\nabla u, \xi\rangle|^{2} \mathrm{~d} x+b \int_{A \cap S(u) \cap E_{0}}\left|\left\langle\nu_{u}, \xi\right\rangle\right| \mathrm{d} \mathcal{H}^{n-1}+\frac{b}{2} \int_{A \cap \partial^{*} E}\left|\left\langle\nu_{E}, \xi\right\rangle\right| \mathrm{d} \mathcal{H}^{n-1} .
$$

Since, if $u \chi_{E_{0}} \in G S B V(A)$, the set function $F^{\prime}(u, E ; \cdot)$ is superadditive on disjoint open sets, an application of Proposition 4 with $\lambda=\mathcal{L}^{n}+\mathcal{H}^{n-1}\left\llcorner\left(S(u) \cap E_{0}\right)+\mathcal{H}^{n-1}\left\llcorner\partial^{*} E\right.\right.$, and

$$
g_{i}(x)= \begin{cases}a\left|\left\langle\nabla u, \xi_{i}\right\rangle\right|^{2} & \text { if } x \in \Omega \backslash\left(\left(S(u) \cap E_{0}\right) \cup \partial^{*} E\right) \\ b\left|\left\langle\nu_{u}, \xi_{i}\right\rangle\right| & \text { if } x \in S(u) \cap E_{0} \\ \frac{b}{2}\left|\left\langle\nu_{E}, \xi_{i}\right\rangle\right| & \text { if } x \in \partial^{*} E\end{cases}
$$

where $\left\{\xi_{i}\right\}$ is a dense sequence in $S^{n-1}$ such that $\left\langle\xi_{i}, \nu_{u}\right\rangle \neq 0 \mathcal{H}^{n-1}$-a.e. on $S(u) \cap E_{0}$, gives

$$
F^{\prime}(u, E ; A) \geq \bar{F}(u, E ; A)
$$

as desired.

Remark 6. The same proof allows to treat the case when $m \geq 1$

$$
F(u, E ; A)= \begin{cases}c \int_{A \backslash E}\|\nabla u\|^{p} \mathrm{~d} x+\int_{\partial E \cap A} \varphi\left(\nu_{E}\right) \mathrm{d} \mathcal{H}^{n-1} & \text { if } u \in W^{1, p}\left(\Omega ; \mathbb{R}^{m}\right), \partial E \text { Lipschitz } \\ +\infty & \text { otherwise }\end{cases}
$$

where $\|\nabla u\|=\sup \{\nabla u \cdot \xi:|\xi|=1\}$ and $c$ is a positive constant, obtaining the lower inequality with $\bar{F}$ as in the thesis of Theorem 1. The necessary modifications to the slicing procedure are standard and can be found in [11], Section 4.1.2.

Remark 7. For general quasiconvex $f$ as in Theorem 1 we may consider the lower semicontinuous functional [5] on $G S B V\left(\Omega ; \mathbb{R}^{m}\right)$ defined by

$$
G(v ; A)=\int_{A} f(\nabla v) \mathrm{d} x+c \mathcal{H}^{n-1}(S(v) \cap A) .
$$


If $u_{j} \rightarrow u$ and $E_{j} \rightarrow E$ we have, setting $v_{j}=u_{j}\left(1-\chi_{E_{j}}\right)$ and $v=u\left(1-\chi_{E}\right)$

$$
\begin{aligned}
\liminf _{j} F\left(u_{j}, E_{j} ; A\right) & \geq \liminf _{j}\left(G\left(v_{j}, A\right)-\left|A \cap E_{j}\right| f(0)\right) \\
& \geq G(v ; A)-|A \cap E| f(0) \\
& =\int_{A} f\left(\left(1-\chi_{E}\right) \nabla u\right) \mathrm{d} x+c \mathcal{H}^{n-1}(S(v) \cap A)-|A \cap E| f(0) \\
& \geq \int_{A \backslash E} f(\nabla u) \mathrm{d} x .
\end{aligned}
$$

Note that this identification of $F$ with $G$ gives a lower bound that is optimal for the bulk term but not for the surface energy.

Remark 8 (Proof of the lower bound in the general case). Let now $F$ be defined as in Theorem 1 and let $u$ and $E$ be such that $u \chi_{E_{0}} \in G S B V\left(\Omega ; \mathbb{R}^{m}\right)$ and $F^{\prime}(u, E ; A)<+\infty$. By the growth conditions on $f$ and Remark 6 we then have

$$
F^{\prime}(u, E ; A) \geq \int_{A \cap \partial^{*} E} \varphi\left(\nu_{E}\right) \mathrm{d} \mathcal{H}^{n-1}+\int_{A \cap S(u) \cap E_{0}}\left(\varphi\left(\nu_{u}\right)+\varphi\left(-\nu_{u}\right)\right) \mathrm{d} \mathcal{H}^{n-1}
$$

by Remark 7 on the other hand we obtain

$$
F^{\prime}(u, E ; A) \geq \int_{A \backslash E} f(\nabla u) \mathrm{d} x .
$$

We can define apply Proposition 4 with $\mu(A)=F^{\prime}(u, E ; A)$, the measure $\lambda$ defined by $\lambda(A)=|A|+\mathcal{H}^{n-1}(A \cap$ $\left.\left(S(u) \cap E_{0}\right)\right)+\mathcal{H}^{n-1}\left(A \cap \partial^{*} E\right), g_{1}(x)=f(\nabla u) \chi_{E_{0} \backslash S(u)}$ and $g_{2}(x)=\varphi\left(\nu_{E}\right) \chi_{\partial^{*} E}+\left(\varphi\left(\nu_{u}\right)+\varphi\left(-\nu_{u}\right)\right) \chi_{S(u) \cap E_{0}}$, to obtain the lower inequality in the general case.

\section{THE UPPER INEQUALITY}

In order to give an upper estimate, we introduce, for every $u \in L^{1}(\Omega), E \in \mathcal{M}(\Omega)$ and $A$ open subset of $\Omega$ :

$$
F^{\prime \prime}(u, E ; A)=\inf \left\{\limsup _{j \rightarrow+\infty} F\left(u_{j}, E_{j} ; A\right): u_{j} \rightarrow u, E_{j} \rightarrow E \text { in } L^{1}(\Omega),\right\} .
$$

The proof of Theorem 2 is complete if we show that the following inequality holds for every $u \in L^{1}(\Omega)$, $E \in \mathcal{M}(\Omega)$ and $A$ open subset of $\Omega$ :

$$
F^{\prime \prime}(u, E ; A) \leq \bar{F}(u, E ; A) .
$$

It is clearly sufficient to prove the following:

Proposition 9. Let $u \in L^{1}(\Omega), E \in \mathcal{M}(\Omega)$ such that $u \chi_{E_{0}} \in G S B V(\Omega)$. Then, there exists a sequence $\left\{\left(u_{j}, E_{j}\right)\right\} \in H^{1}(\Omega) \times \mathcal{M}(\Omega)$, with $\partial E_{j}$ of class $C^{\infty}$, such that $u_{j} \rightarrow u, E_{j} \rightarrow E$ in $L^{1}(\Omega)$, and

$$
\limsup _{j \rightarrow+\infty} F\left(u_{j}, E_{j}\right) \leq \bar{F}(u, E) .
$$

In order to construct the recovery sequence, let us recall the definition of strong convergence in $S B V^{p}$, introduced in [14], and an approximation lemma for $S B V^{p}$ with piecewise $C^{1}$ functions. 
Definition 10. ([14]) Let $\left\{u_{j}\right\}$ be a sequence of functions in $S B V^{p}$. We say that $u_{j}$ converges strongly to $u$ in $S B V^{p}$ if

$-u_{j} \rightarrow u$ in $L^{1}(\Omega)$,

- $\nabla u_{j} \rightarrow \nabla u$ strongly in $L^{p}\left(\Omega ; \mathbb{R}^{n}\right)$,

$-\mathcal{H}^{n-1}\left(S\left(u_{j}\right) \Delta S(u)\right) \rightarrow 0$,

$-\int_{S\left(u_{j}\right) \cup S(u)}\left(\left|u_{j}^{+}-u^{+}\right|+\left|u_{j}^{-}-u^{-}\right|\right) \mathrm{d} \mathcal{H}^{n-1} \rightarrow 0$

(we choose the orientation $\nu_{u_{j}}=\nu_{u} \mathcal{H}^{n-1}$-a.e. on $S\left(u_{j}\right) \cap S(u)$; recall that if $v \in B V(\Omega)$ then we set $v^{+}=v^{-}=\widetilde{v}$ on $\Omega \backslash S(v))$.

Lemma 11. [14] If $u \in S B V^{p}(\Omega) \cap L^{\infty}(\Omega)$ then there exists a sequence $\left\{u_{j}\right\}$ in $S B V^{p}(\Omega) \cap L^{\infty}(\Omega)$ with $\left\|u_{j}\right\|_{\infty} \leq\|u\|_{\infty}$, strongly converging to $u$ in $S B V^{p}$, such that for each $j \in \mathbb{N}$ there exists a closed rectifiable set $R_{j}$ such that $u_{j} \in C^{1}\left(\Omega \backslash R_{j}\right)$. Moreover, $R_{j}$ can be chosen so that its Minkowski content coincides with $\mathcal{H}^{n-1}\left(R_{j}\right) ;$ i.e.,

$$
\mathcal{H}^{n-1}\left(R_{j}\right)=\lim _{\rho \rightarrow 0^{+}} \frac{1}{2 \rho}\left|\left\{x: \operatorname{dist}\left(x, R_{j}\right)<\rho\right\}\right| .
$$

The proof of this Lemma in [14] consists in finding first a compact set $K \subset S(u)$ such that $\mathcal{H}^{n-1}(S(u) \backslash K) \ll 1$ (which will be the main part of $R_{j}$ ) and then then approximating $u$ on $\Omega \backslash K$ by minimizing a Mumford-Shah type functional.

In the following $M^{n-1}(B)$ stands for the Minkowski content of a set $B$. As an intermediate step in the construction of the recovery sequence $\left\{\left(u_{j}, E_{j}\right)\right\}$, we apply the approximation result of Lemma 11 to prove the following:

Lemma 12. Let $E$ be a set of finite perimeter with $E=\Omega \backslash E_{0}$ and $u \in L^{\infty}(\Omega)$, such that $u \chi_{E_{0}} \in S B V(\Omega)$. Then, there exist a sequence of closed rectifiable sets $\left\{S_{j}\right\}$, a sequence of measurable subsets $\left\{F_{j}\right\}$, with $\partial F_{j}$ closed rectifiable and Lipschitz in $\Omega \backslash S_{j}$, and a sequence of functions $\left\{w_{j}\right\}$ in $S B V(\Omega) \cap C^{1}\left(\Omega \backslash\left(S_{j} \cup \partial F_{j}\right)\right)$ such that

$$
\begin{aligned}
& -w_{j} \rightarrow u \text { and } F_{j} \rightarrow E \text { in } L^{1}(\Omega) ; \\
& -\mathcal{H}^{n-1}\left(S_{j} \backslash\left(S\left(w_{j}\right) \cap\left(F_{j}\right)_{0}\right)\right)=o(1)_{j \rightarrow+\infty} ; \\
& -\mathcal{H}^{n-1}\left(\partial F_{j}\right)=M^{n-1}\left(\partial F_{j}\right), \quad \mathcal{H}^{n-1}\left(S_{j}\right)=M^{n-1}\left(S_{j}\right) ; \\
& -\limsup _{j \rightarrow+\infty} \bar{F}\left(w_{j}, F_{j}\right) \leq \bar{F}(u, E) .
\end{aligned}
$$

Proof. We can suppose $\|u\|_{\infty}=1$; we set

$$
\bar{u}:=\left\{\begin{array}{lll}
u & \text { in } \Omega \backslash E \\
2 & \text { in } E
\end{array}\right.
$$

From Lemma 11, for every $j$ there exist a closed rectifiable set $R_{j}$ and a function $\bar{u}_{j} \in C^{1}\left(\Omega \backslash R_{j}\right)$, such that $\bar{u}_{j}$ strongly converges to $\bar{u}$, and $\mathcal{H}^{n-1}\left(R_{j} \backslash S\left(\bar{u}_{j}\right)\right) \rightarrow 0$.

Setting $v_{j}=\left(\bar{u}_{j} \vee 1\right) \wedge 2$ and $v=(\bar{u} \vee 1) \wedge 2$, it follows that the sequence $\left\{v_{j}\right\}$ strongly converges to $v$; in particular,

$$
\left|D v_{j}\right|(\Omega)=\int_{\Omega}\left|\nabla v_{j}\right| \mathrm{d} x+\int_{S\left(v_{j}\right)}\left|v_{j}^{+}-v_{j}^{-}\right| \mathrm{d} \mathcal{H}^{n-1} \rightarrow \int_{\Omega}|\nabla v| \mathrm{d} x+\int_{S(v)}\left|v^{+}-v^{-}\right| \mathrm{d} \mathcal{H}^{n-1},
$$

and

$$
|D v|(\Omega)=\mathcal{H}^{n-1}(S(v))=\mathcal{H}^{n-1}\left(\partial^{*} E\right) .
$$

The coarea formula gives, for $A$ open subset of $\Omega$ :

$$
\mathcal{H}^{n-1}\left(\partial^{*} E \cap A\right)=\lim _{j \rightarrow+\infty} \int_{1}^{2} \mathcal{H}^{n-1}\left(\partial^{*} E_{j}^{t} \cap A\right) \mathrm{d} t
$$


where $E_{j}^{t}=\left\{x \in A: \bar{u}_{j} \geq t\right\}$. Fixing $\delta>0$, there exists $t_{j} \in(1,2-\delta)$ such that, setting $F_{j}=E_{j}^{t_{j}}$,

$$
\mathcal{H}^{n-1}\left(\partial F_{j} \cap A\right) \leq \frac{1}{1-\delta} \mathcal{H}^{n-1}(\partial E \cap A)
$$

and $\partial F_{j}$ is Lipschitz in $\Omega \backslash R_{j}$.

Now, we define the sequence $\left\{w_{j}\right\}$ as:

$$
w_{j}:=\bar{u}_{j} \chi_{\left(F_{j}\right)_{0}}
$$

it follows that $w_{j} \in C^{1}\left(\Omega \backslash\left(R_{j} \cup \partial F_{j}\right)\right)$, and setting $S_{j}=\overline{R_{j} \cap\left(F_{j}\right)_{0}}$ we get

$$
\mathcal{H}^{n-1}\left(S_{j} \backslash\left(S\left(w_{j}\right) \cap\left(E_{j}\right)_{0}\right)\right)=o(1)_{j \rightarrow+\infty} .
$$

Moreover:

$$
\int_{\Omega}|\nabla u|^{2} \mathrm{~d} x \leq \liminf _{j \rightarrow+\infty} \int_{\Omega}\left|\nabla w_{j}\right|^{2} \mathrm{~d} x \leq \liminf _{j \rightarrow+\infty} \int_{\Omega}\left|\nabla \bar{u}_{j}\right|^{2} \mathrm{~d} x=\int_{\Omega}|\nabla \bar{u}|^{2} \mathrm{~d} x=\int_{\Omega}|\nabla u|^{2} \mathrm{~d} x,
$$

and this implies the convergence

$$
\nabla w_{j} \rightarrow \nabla u \quad \text { in } L^{2}(\Omega)
$$

The strong convergence $\bar{u}_{j} \rightarrow \bar{u}$ entails:

$$
\begin{aligned}
\mathcal{H}^{n-1}(\partial E)+\mathcal{H}^{n-1}(S(\bar{u}) \backslash E)=\mathcal{H}^{n-1}(S(\bar{u})) & =\lim _{j \rightarrow+\infty} \mathcal{H}^{n-1}\left(S\left(\bar{u}_{j}\right)\right) \\
& =\lim _{j \rightarrow+\infty}\left(\mathcal{H}^{n-1}\left(S\left(\bar{u}_{j}\right) \backslash F_{j}\right)+\mathcal{H}^{n-1}\left(S\left(\bar{u}_{j}\right) \cap F_{j}\right)\right) \\
& =\lim _{j \rightarrow+\infty}\left(\mathcal{H}^{n-1}\left(S\left(w_{j}\right) \backslash F_{j}\right)+\mathcal{H}^{n-1}\left(S\left(\bar{u}_{j}\right) \cap F_{j}\right)\right) .
\end{aligned}
$$

Again from the strong convergence $\bar{u}_{j} \rightarrow \bar{u}$ we obtain, in particular:

$$
\lim _{j \rightarrow+\infty} \int_{\partial E \backslash\left(S\left(\bar{u}_{j}\right) \cap F_{h}\right)}\left(\left|1-\left(\bar{u}_{j}\right)^{-}\right|+\left|2-\left(\bar{u}_{j}\right)^{+}\right|\right) \mathrm{d} \mathcal{H}^{n-1}=0 ;
$$

since $\left|1-\left(\bar{u}_{j}\right)^{-}\right|+\left|2-\left(\bar{u}_{j}\right)^{+}\right| \geq \delta$ in $\partial E \backslash\left(S\left(\bar{u}_{j}\right) \cap F_{j}\right)$, it follows that

$$
\lim _{j \rightarrow+\infty} \mathcal{H}^{n-1}\left(\partial E \backslash\left(S\left(\bar{u}_{j}\right) \cap F_{j}\right)\right)=0 .
$$

Then, we obtain

$$
\begin{aligned}
\mathcal{H}^{n-1}(\partial E) & =\lim _{j \rightarrow+\infty} \mathcal{H}^{n-1}\left(\partial E \cap\left(S\left(\bar{u}_{j}\right) \cap F_{j}\right)\right) \\
& \leq \liminf _{j \rightarrow+\infty} \mathcal{H}^{n-1}\left(S\left(\bar{u}_{j}\right) \cap F_{j}\right) .
\end{aligned}
$$

This inequality, taking into account (8), allows to deduce that:

$$
\limsup _{j \rightarrow+\infty} \mathcal{H}^{n-1}\left(S\left(w_{j}\right) \backslash F_{j}\right) \leq \mathcal{H}^{n-1}(S(\bar{u}) \backslash E)=\mathcal{H}^{n-1}(S(u) \backslash E),
$$

concluding the proof of the lemma. 
Proof of Proposition 9. To prove Proposition 9, we consider a function $u$ and a set $E$ such that $\bar{F}(u, E)$ is finite. A truncation argument allows us to suppose $u \in L^{\infty}(\Omega)$. Now, we consider the sequences $\left\{w_{j}\right\},\left\{F_{j}\right\}$ and $\left\{S_{j}\right\}$ given by Lemma 12. From the coarea formula, recalling that $\mathcal{H}^{n-1}\left(S_{j}\right)=M^{n-1}\left(S_{j}\right)$ and $\left|\nabla \operatorname{dist}\left(\cdot, S_{j}\right)\right|=1$ a.e., for fixed $j$ it follows:

$$
\begin{aligned}
k \int_{0}^{1 / k} \mathcal{H}^{n-1}\left(\partial^{*}\left\{x \in \Omega: \operatorname{dist}\left(x, S_{j}\right)<r\right\}\right) \mathrm{d} r & =\int_{\left\{\operatorname{dist}\left(\cdot, S_{j}\right)<1 / k\right\}}\left|\nabla \operatorname{dist}\left(x, S_{j}\right)\right| \mathrm{d} x \\
& =k\left|\left\{x \in \Omega: \operatorname{dist}\left(x, S_{j}\right)<\frac{1}{k}\right\}\right|=2 \mathcal{H}^{n-1}\left(S_{j}\right)+o(1)_{k \rightarrow+\infty}
\end{aligned}
$$

so that there exists $r_{j}^{k} \in(0,1 / k)$ with, letting $\Sigma_{j}^{k}=\left\{x \in \Omega: \operatorname{dist}\left(x, S_{j}\right)<r_{j}^{k}\right\}$,

$$
\mathcal{H}^{n-1}\left(\partial \Sigma_{j}^{k}\right) \leq 2 \mathcal{H}^{n-1}\left(S_{j}\right)+o(1)_{k \rightarrow+\infty}
$$

Upon choosing a suitable sequence $k_{j}$ and defining $\Sigma_{j}=\Sigma_{j}^{k_{j}}$, we then have

$$
\mathcal{H}^{n-1}\left(\partial \Sigma_{j}\right) \leq 2 \mathcal{H}^{n-1}\left(S_{j}\right)+o(1)_{j \rightarrow+\infty}
$$

Now, setting

$$
E_{j}=F_{j} \cup \Sigma_{j},
$$

since $\mathcal{H}^{n-1}\left(S_{j}\right)=\mathcal{H}^{n-1}\left(S\left(w_{j}\right) \cap E_{0}\right)+o(1)_{j \rightarrow+\infty}$, we get:

$$
\mathcal{H}^{n-1}\left(\partial E_{j}\right) \leq \mathcal{H}^{n-1}\left(\partial F_{j}\right)+2 \mathcal{H}^{n-1}\left(S\left(w_{j}\right)\right)+o(1)_{j \rightarrow+\infty}
$$

For every $E_{j}$, it is easy to show that there exists a set $E_{j}^{\prime}$ of class $C^{\infty}$ such that $E_{j} \subset E_{j}^{\prime}$ and $\mathcal{H}^{n-1}\left(\partial E_{j}^{\prime}\right)=$ $\mathcal{H}^{n-1}\left(\partial E_{j}\right)+o(1)$, so we can assume $E_{j}$ of class $C^{\infty}$. Then we can find, for every $j$, a function $\tilde{u}_{j} \in H^{1}(\Omega)$ such that the restriction of $u_{j}$ to the set $\left(E_{j}\right)_{0}$ coincides with the restriction of $w_{j}$. We then set $u_{j}=\phi_{j} \tilde{u}_{j}+\left(1-\phi_{j}\right) v_{j}$, where $v_{j}$ are smooth functions converging to $u$ in $L^{1}(\Omega)$, and $\phi_{j}$ are smooth functions with $\phi_{j}=1$ on $\left(E_{j}\right)_{0}$ and $\phi_{j}(x)=0$ if $\operatorname{dist}\left(x,\left(E_{j}\right)_{0}\right)>1 /\left(2 r_{j}\right)$. Clearly, the sequence $\left\{u_{j}\right\}$ converges to $u$ in $L^{1}(\Omega)$; the inequality (11) implies:

$$
\limsup _{j \rightarrow+\infty} F\left(u_{j}, E_{j}\right) \leq \limsup _{j \rightarrow+\infty} \bar{F}\left(w_{j}, E_{j}\right),
$$

and this completes the proof of the upper inequality and of the Proposition 9.

Remark 13. An alternative proof of the upper inequality would consist in first approximating the set $E$ as follows: since $\chi_{E} \in B V(\Omega)$, by standard results [21] there exists a sequence $\left\{v_{j}\right\} \subset C^{\infty}(\Omega)$, converging to $\chi_{E}$ in $L^{1}(\Omega)$ and such that $\int_{\Omega}\left|\nabla v_{j}\right| \rightarrow\left|D \chi_{E}\right|=\mathcal{H}^{n-1}\left(\partial^{*} E\right)$. It is easy to show that for all $s \in(0,1), \chi_{\left\{v_{j} \geq s\right\}} \rightarrow \chi_{E}$ in $L^{1}(\Omega)$.

Moreover, for a.a. $s \in(0,1), \partial\left\{v_{j} \geq s\right\} \cap \Omega$ is $C^{\infty}$ (by Sard's lemma), and using the coarea formula we find

$$
\mathcal{H}^{n-1}\left(\partial\left\{v_{j} \geq s\right\} \cap \Omega\right) \rightarrow \mathcal{H}^{n-1}\left(\partial^{*} E\right) .
$$

From the construction in [21] (made by locally convolving $\chi_{E}$ with suitable mollifiers) we also can assume that

$$
v_{j}(x) \rightarrow\left\{\begin{array}{lll}
0 & \text { in } E_{0} \\
1 & \text { in } E_{1} \\
\frac{1}{2} & \text { in } E_{1 / 2}
\end{array} \quad \mathcal{H}^{n-1} \text {-a.a. } x\right.
$$


where $E_{1 / 2}$ is the set of points where $E$ has density $\frac{1}{2}$. Hence, for $s<\frac{1}{2}$, it follows that:

$$
\chi_{\left\{v_{j} \geq s\right\}}(x) \rightarrow\left\{\begin{array}{lll}
0 & \text { in } E_{0} \\
1 & \text { in } \Omega \backslash E_{0},
\end{array} \mathcal{H}^{n-1} \text {-a.a. } x\right.
$$

recalling that $\mathcal{H}^{n-1}\left(\Omega \backslash\left(E_{0} \cup E_{1} \cup E_{1 / 2}\right)\right)=0$; in particular, for $s<\frac{1}{2}$ :

$$
\int_{\partial^{*} E} \chi_{\left\{v_{j} \geq s\right\}}(x) \mathrm{d} \mathcal{H}^{n-1} \rightarrow \mathcal{H}^{n-1}\left(\partial^{*} E\right) .
$$

Then, for $\varepsilon>0$, we can choose $s<\frac{1}{2}$ such that, setting $F_{j}=\left\{v_{j} \geq s\right\}$ :

$$
\mathcal{H}^{n-1}\left(\partial^{*} E \backslash F_{j}\right)<\varepsilon \quad \text { for } j \text { large enough. }
$$

Now, let $u \in S B V(\Omega)$. We let $u_{j}^{\prime}=\left.u\right|_{\Omega \backslash F_{j}}$, which is viewed as a function defined in the open set $\Omega \backslash F_{j}$. Clearly $u^{\prime} \in S B V\left(\Omega \backslash F_{j}\right)$, and one has

$$
S\left(u_{j}^{\prime}\right) \subset\left(\partial^{*} E \backslash F_{j}\right) \cup\left(S(u) \cap E_{0}\right),
$$

so that

$$
\mathcal{H}^{n-1}\left(S\left(u_{j}^{\prime}\right)\right) \leq \mathcal{H}^{n-1}\left(S(u) \cap E_{0}\right)+\varepsilon .
$$

By standard approximation results, for every $j$ there exists $u_{j} \in S B V\left(\Omega \backslash F_{j}\right)$ with $\left\|u_{j}-u_{j}^{\prime}\right\|_{L^{1}}<1 / j$, and such that: $\mathcal{H}^{n-1}\left(\overline{S\left(u_{j}\right)} \backslash S\left(u_{j}\right)\right)=0$ (so that $\mathcal{H}^{n-1}\left(S\left(u_{j}\right)\right)=M^{n-1}\left(S\left(u_{j}\right)\right)$ ), $u_{j} \in C^{1}\left(\left(\Omega \backslash F_{j}\right) \backslash \overline{S\left(u_{j}\right)}\right.$ ), and

$$
\mathcal{H}^{n-1}\left(S\left(u_{j}\right)\right) \leq \mathcal{H}^{n-1}\left(S\left(u_{j}^{\prime}\right)\right)+\varepsilon
$$

Recalling (12), this inequality and (13), we find:

$$
\mathcal{H}^{n-1}\left(S\left(u_{j}\right)\right) \leq \mathcal{H}^{n-1}\left(S(u) \cap E_{0}\right)+2 \varepsilon
$$

We can now select an appropriate neighborhood $\Sigma_{j}$ of $S_{j}=S\left(u_{j}\right)$ as in the previous proof, and letting $E_{j}=$ $F_{j} \cup \Sigma_{j}$, we conclude as before.

Remark 14 (Proof of the upper bound in the general case). The proof is not much modified in the general case since the main difficulty is the construction of the approximating sets, which is independent of $m$ and of the particular energy.

Some care must be taken while following the reasoning in (10). In the case when $\varphi$ is even, then standard results on the anisotropic Minkowski contents (where the distance function is replaced by $\varphi^{\circ}: x \mapsto \sup _{\varphi(\xi) \leq 1}$ $\xi \cdot x$, the polar of $\varphi$, see [6]) allow to easily adapt the previous proof to the anisotropic case. However, the nonsymmetric case is not covered by these results (although it is very likely that they extend to this situation).

We first note that if $S_{j}$ is composed of a finite number of compact subsets of $C^{1}$ hypersurfaces, by the condition $M^{n-1}\left(S_{j}\right)=\mathcal{H}^{n-1}\left(S_{j}\right)$ we have

$$
k\left|\left\{x \in \Omega: \operatorname{dist}\left(x, S_{j}\right)<\frac{1}{k}\right\}\right|=k\left|\left\{y+t \nu(y): y \in S_{j},|t|<\frac{1}{k}\right\}\right|+o(1)_{k \rightarrow+\infty}
$$


(but in the second representation, the same point might correspond to two or more values of $y$ and $t$ ). We then have

$$
\begin{aligned}
k & \int_{0}^{1 / k} \int_{\partial^{*}\left\{x \in \Omega: \operatorname{dist}\left(x, S_{j}\right)<r\right\}} \varphi\left(\nabla \operatorname{dist}\left(x, S_{j}\right)\right) \mathrm{d} \mathcal{H}^{n-1} \\
& =\int_{\left\{\operatorname{dist}\left(\cdot, S_{j}\right)<1 / k\right\}} \varphi\left(\nabla \operatorname{dist}\left(x, S_{j}\right)\right)\left|\nabla \operatorname{dist}\left(x, S_{j}\right)\right| \mathrm{d} x \\
& \leq k \int_{S_{j}} \int_{-1 / k}^{1 / k} \varphi\left(\nabla \operatorname{dist}\left(y+t \nu(y), S_{j}\right)\right) \mathrm{d} t \mathrm{~d} \mathcal{H}^{n-1}(y)+o(1)_{k \rightarrow+\infty} \\
& =\int_{S_{j}}(\varphi(\nu)+\varphi(-\nu)) \mathrm{d} \mathcal{H}^{n-1}+o(1)_{k \rightarrow+\infty}
\end{aligned}
$$

since $\mathcal{H}^{n-1}$-a.e. on $S_{j}$

$$
\lim _{t \rightarrow 0^{ \pm}} \nabla \operatorname{dist}\left(y+t \nu(y), S_{j}\right)= \pm \nu(y) .
$$

If we define $\Sigma_{j}^{k}$ as before, we find that

$$
\limsup _{k \rightarrow \infty} \int_{\partial^{*} \Sigma_{j}^{k}} \varphi\left(\nabla \operatorname{dist}\left(x, S_{j}\right)\right) \mathrm{d} \mathcal{H}^{n-1}=\limsup _{k \rightarrow \infty} \int_{\partial^{*} \Sigma_{j}^{k}} \varphi\left(\nu_{\Sigma_{j}^{k}}\right) \mathrm{d} \mathcal{H}^{n-1} \leq \int_{S_{j}}(\varphi(\nu)+\varphi(-\nu)) \mathrm{d} \mathcal{H}^{n-1}
$$

and are able to carry on the proof of Proposition 9. In the general case we can split $S_{j}$ in a part composed of a finite union of compact subsets of $C^{1}$ hypersurfaces and a remainder whose Minkowski content is arbitrarily small, and proceed likewise.

The only technical point where some extra care must be used is the truncation argument at the beginning of the proof of Proposition 9. That argument is straightforward in the scalar case while in the vector case some more elaborate but by now standard truncation lemmas must be used (for example [17], Lem. 3.5).

It remains to prove that if $0<|E| \leq \Omega$ then we can find a recovery sequence with $\left|E_{j}\right|=|E|$. The case $|E|=\Omega$ is trivial. In the case $0<|E|<\Omega$ we can simply modify $E_{j}$ by inserting or removing suitable balls with suitable volume close to ||$E|-| E_{j}||$ (and possibly smoothing the resulting sets if needed). The location of the centres of such balls must be chosen as a point of density 0 or 1 for $E$, respectively. Details can be found, e.g., in [2], Theorem 3.3.

Remark 15 (An extension). As suggested by an anonymous referee, the results of Theorem 1 can be extended to a more general $F$ of the form

$$
F(u, E)=\int_{\Omega \backslash E} f(\nabla u) \mathrm{d} x+\int_{\partial E} \varphi\left(u^{-}, \nu_{E}\right) \mathrm{d} \mathcal{H}^{n-1},
$$

where $u^{-}$denotes the trace of the restriction of $u$ to $\Omega \backslash E$ on $\partial E$. If $\varphi: \Omega \times \mathbb{R}^{n} \rightarrow[0,+\infty)$ is a continuous function such that $\varphi(u, \cdot)$ is convex and positively homogeneous of degree one and $\varphi(u, z) \geq c|z|$ with $c>0$, then the thesis of Theorem 1 still holds with

$$
F(u, E)=\int_{\Omega \backslash E} f(\nabla u) \mathrm{d} x+\int_{\partial^{*} E} \varphi\left(u^{-}, \nu_{E}\right) \mathrm{d} \mathcal{H}^{n-1}+\int_{\Omega \cap S(u) \cap E_{0}}\left(\varphi\left(u^{-}, \nu_{u}\right)+\varphi\left(u^{+},-\nu_{u}\right)\right) \mathrm{d} \mathcal{H}^{n-1},
$$

with the usual notation for the traces of $u$ on both sides of $S(u)$.

The proof of the lower bound can be achieved in the same way, noting that the lower bound for the bulk part (Rem. 7) remains unchanged, while we first easily deal with one-dimensional integrands of the form $\varphi\left(u^{-}\right)$, subsequently obtaining the lower bound by slicing. In view of Remark 7 is is worth noting that, even in the 
simplest case $\varphi=\varphi\left(u^{-}\right)$, a necessary condition for the lower semicontinuity of the functional

$$
G(v ; A)=\int_{A} f(\nabla v) \mathrm{d} x+\int_{S(v) \cap A} \varphi\left(v^{+}-v^{-}\right) \mathrm{d} \mathcal{H}^{n-1}
$$

(which is the analog of the $G$ in that remark) is that $\varphi$ is subadditive, while in our case the jump energy density on $S(u)$ turns out to be $\psi\left(u^{+}, u^{-}\right)=\varphi\left(u^{+}\right)+\varphi\left(u^{-}\right)$, which is automatically subadditive. The proof of the upper bound holds with technical changes that are left to the interested reader.

\section{Applications}

\subsection{An approximation of the Mumford-Shah functional}

As a byproduct of Theorem 2 we have an approximation of the Mumford-Shah functional by energies defined on pairs function-set defined as follows. Let $r_{\varepsilon}$ be a family of strictly positive numbers converging to 0 ; for every $\varepsilon>0$ we set

with $F$ defined by (1).

$$
H_{\varepsilon}(u, E)= \begin{cases}F(u, E) & \text { if }|E| \leq r_{\varepsilon} \\ +\infty & \text { otherwise }\end{cases}
$$

Theorem 16. The functionals $H_{\varepsilon} \Gamma$-converge to the energy (equivalent to the Mumford-Shah functional)

$$
H(u, E)= \begin{cases}a \int_{\Omega}|\nabla u|^{2}+b \mathcal{H}^{n-1}(S(u)) & \text { if } u \in G S B V(\Omega),|E|=0 \\ +\infty & \text { otherwise. }\end{cases}
$$

Proof. Note that if $\liminf _{\varepsilon \rightarrow 0} H_{\varepsilon}\left(u_{\varepsilon}, E_{\varepsilon}\right)$ then $\left|E_{\varepsilon}\right| \rightarrow 0$ and hence we can limit our analysis to $|E|=0$. The lower inequality immediately follows by applying Theorem 2, and noticing that for such $E$ we have $E_{0}=\Omega$. The same inequality implies that $u \in G S B V(\Omega)$.

Conversely, again by Theorem 2 we have that for every $u \in G S B V(\Omega)$ we can find sets $E_{j}$ with $\left|E_{j}\right| \rightarrow 0$ and functions $u_{j}$ such that $H(u, E)=\lim _{j} F\left(u_{j}, E_{j}\right)$. For fixed $\left\{\varepsilon_{j}\right\}$, upon extracting a subsequence of $E_{j}$, we can always suppose that $\left|E_{j}\right| \leq r_{\varepsilon_{j}}$, so that $H(u, E)=\lim _{j} H_{\varepsilon_{j}}\left(u_{j}, E_{j}\right)$. By the arbitrariness of $\left\{\varepsilon_{j}\right\}$ the upper bound is proved.

\subsection{A generalization of the Ambrosio and Tortorelli approximation result}

In this paragraph we use Theorem 16 to give a proof of the Ambrosio-Tortorelli result [4]. We take the chance for a slight generalization.

Let $V:[0,1] \rightarrow[0,+\infty)$ be a continuous function vanishing at the point 1 and strictly positive on $(0,1)$, and let $V_{\varepsilon}:[0,1] \rightarrow[0,+\infty)$ be continuous functions converging uniformly to $V$ such that $V_{\varepsilon}(1)=0, V_{\varepsilon}>0$ on $[0,1)$ and there exist $K_{\varepsilon} \rightarrow 0$ such that $\varepsilon<<K_{\varepsilon}$ and a neighbourhood $[0, \eta]$ of 0 independent of $\varepsilon$ where $V_{\varepsilon} \geq K_{\varepsilon}$. The prototype situation is choosing $V$ vanishing also in 0 (a 'double-well potential') and $V_{\varepsilon}(z)=V(z)+\sqrt{\varepsilon}(1-z)$.

For every $\varepsilon>0$, we consider the functional $G_{\varepsilon}: L^{1}(\Omega) \times L^{1}(\Omega) \rightarrow[0,+\infty]$ defined by:

$$
G_{\varepsilon}(u, v)= \begin{cases}\int_{\Omega}\left(v^{2}|\nabla u|^{2}+\frac{V_{\varepsilon}(v)}{\varepsilon}+\varepsilon|\nabla v|^{2}\right) \mathrm{d} x & \text { if } u, v \in H^{1}(\Omega) \text { and } 0 \leq v \leq 1 \text { a.e. } \\ +\infty & \text { otherwise. }\end{cases}
$$

For $u, v \in L^{1}(\Omega)$, we denote by $G^{\prime}(u, v)$ the functional

$$
\Gamma-\liminf _{\varepsilon \rightarrow 0} G_{\varepsilon}(u, v)=\inf \left\{\liminf _{j \rightarrow \infty} G_{\varepsilon_{j}}\left(u_{j}, v_{j}\right): \varepsilon_{j} \rightarrow 0, u_{j} \rightarrow u, v_{j} \rightarrow v \text { in } L^{1}(\Omega)\right\} .
$$


Theorem 17. For every $(u, v) \in L^{1}(\Omega) \times L^{1}(\Omega)$ :

$$
G^{\prime}(u, v) \geq G(u, v)
$$

where

$$
G(u, v)= \begin{cases}\int_{\Omega}|\nabla u|^{2} \mathrm{~d} x+4 \int_{0}^{1} \sqrt{V(s)} \mathrm{d} s \mathcal{H}^{n-1}(S(u)) & \text { if } u \in G S B V(\Omega) \\ +\infty & \text { and } v=1 \text { a.e. }\end{cases}
$$

Proof of Theorem 17. We prove that, if $G^{\prime}(u, v)$ is finite, then $v=1$ a.e., $u \in G S B V(\Omega)$ and

$$
G^{\prime}(u, v) \geq \int_{\Omega}|\nabla u|^{2} \mathrm{~d} x+4 \int_{0}^{1} \sqrt{V(s)} \mathrm{d} s \mathcal{H}^{n-1}(S(u)) .
$$

Assume $G^{\prime}(u, v)<+\infty$, and $u_{\varepsilon} \rightarrow u, v_{\varepsilon} \rightarrow v$ be such that $G_{\varepsilon}\left(u_{\varepsilon}, v_{\varepsilon}\right) \leq c<+\infty$. Then

$$
\left|\left\{v_{\varepsilon} \leq \eta\right\}\right| \leq \frac{\varepsilon}{K_{\varepsilon}} G_{\varepsilon}\left(u_{\varepsilon}, v_{\varepsilon}\right)=o(1)
$$

implies that $v \geq \eta$ in measure. Since $V_{\varepsilon} \rightarrow V$ uniformly, and $V(z)$ vanishes only at the point 1 for $z \geq \eta$, we deduce that $v=1$ a.e.

We introduce, for every $A$ open subset of $\Omega$, the localized functionals:

$$
G_{\varepsilon}(u, v ; A)= \begin{cases}\int_{A}\left(v^{2}|\nabla u|^{2}+\frac{V_{\varepsilon}(v)}{\varepsilon}+\varepsilon|\nabla v|^{2}\right) \mathrm{d} x & \text { if } u, v \in H^{1}(\Omega), \\ +\infty & \text { and } 0 \leq v \leq 1 \text { a.e. }\end{cases}
$$

Clearly, since $G^{\prime}(u, v)<+\infty$, it follows that $G^{\prime}(u, v ; A):=\Gamma-\liminf _{\varepsilon \rightarrow 0} G(u, v ; A)<+\infty$ for every $A$.

Let $\left\{\varepsilon_{j}\right\}$ be a positive infinitesimal sequence, and $\left\{u_{j}\right\},\left\{v_{j}\right\}$ sequences in $H^{1}(\Omega)$ respectively converging in $L^{1}(\Omega)$ to $u$ and $v$, with $0 \leq v_{j} \leq 1$ a.e. An application of the coarea formula gives:

$$
\begin{aligned}
G_{\varepsilon_{j}}\left(u_{j}, v_{j} ; A\right) & \geq \int_{A}\left|\nabla u_{j}\right|^{2} \mathrm{~d} x+2 \int_{A}\left(\sqrt{V\left(v_{j}\right)}+o(1)\right)\left|\nabla v_{j}\right| \mathrm{d} x \\
& \geq \int_{A}\left|\nabla u_{j}\right|^{2} \mathrm{~d} x+2 \int_{0}^{1}\left(\sqrt{V\left(v_{j}\right)}+o(1)\right) \mathcal{H}^{n-1}\left(\partial\left\{v_{j}<s\right\} \cap A\right) \mathrm{d} s
\end{aligned}
$$

Now, we fix $\delta \in(0,1)$. The Mean Value Theorem ensures the existence of $t_{j}^{\delta} \in(\delta, 1)$ such that

$$
\int_{\delta}^{1} \sqrt{V(s)} \mathcal{H}^{n-1}\left(\partial\left\{v_{j}<s\right\} \cap A\right) \mathrm{d} s \geq \int_{\delta}^{1} \sqrt{V(s)} \mathrm{d} s \mathcal{H}^{n-1}\left(\partial E_{j}^{\delta} \cap A\right)
$$

where $E_{j}^{\delta}=\left\{v_{j}<t_{j}^{\delta}\right\}$; hence

$$
G_{\varepsilon_{j}}\left(u_{j}, v_{j} ; A\right) \geq \delta^{2} \int_{A \backslash E_{j}^{\delta}}\left|\nabla u_{j}\right|^{2} \mathrm{~d} x+2 \int_{\delta}^{1}(\sqrt{V(s)}+o(1)) \mathrm{d} s \mathcal{H}^{n-1}\left(\partial E_{j}^{\delta} \cap A\right) .
$$


Since $v=1$ a.e. in $\Omega$, it follows that $\left|E_{j}^{\delta}\right| \rightarrow 0$ as $j \rightarrow+\infty$, for every $\delta$. An application of Theorem 16 with $a=\delta^{2}$ and $b=4 \int_{\delta}^{1} \sqrt{V(s)} \mathrm{d} s$ allows to deduce that $u \in G S B V(\Omega)$; moreover,

$\liminf _{j \rightarrow+\infty}\left(\delta^{2} \int_{A \backslash E_{j}^{\delta}}\left|\nabla u_{j}\right|^{2} \mathrm{~d} x+2 \int_{\delta}^{1} \sqrt{V(s)} \mathrm{d} s \mathcal{H}^{n-1}\left(\partial E_{j}^{\delta} \cap A\right)\right) \geq \delta^{2} \int_{A}|\nabla u|^{2} \mathrm{~d} x+4 \int_{\delta}^{1} \sqrt{V(s)} \mathrm{d} s \mathcal{H}^{n-1}(S(u) \cap A)$.

Therefore, recalling (15), for every $\delta \in(0,1)$ we get:

$$
\liminf _{j \rightarrow+\infty} G_{\varepsilon_{j}}\left(u_{j}, v_{j} ; A\right) \geq \delta^{2} \int_{A}|\nabla u|^{2} \mathrm{~d} x+4 \int_{\delta}^{1} \sqrt{V(s)} \mathrm{d} s \mathcal{H}^{n-1}(S(u) \cap A) .
$$

In order to apply Proposition 4 to the set function

$$
\mu(A)=\Gamma-\liminf _{\varepsilon \rightarrow 0} G_{\varepsilon}(u, v ; A),
$$

which is superadditive on disjoint open sets when $u \in G S B V(\Omega)$ and $v=1$ a.e., we define:

$$
g_{i}(x)=\left\{\begin{array}{lll}
\delta_{i}^{2}|\nabla u|^{2} & \text { if } & x \in \Omega \backslash S(u) \\
4 \int_{\delta_{i}}^{1} \sqrt{W(s)} \mathrm{d} s & \text { if } \quad x \in S(u)
\end{array}\right.
$$

where $\left\{\delta_{i}\right\}$ is a dense sequence in $(0,1)$, and $\lambda=\mathcal{L}^{n}+\mathcal{H}^{n-1}\left\llcorner S(u)\right.$. From (16) we have $\mu(A) \geq \sup _{i} \int_{A} g_{i} \mathrm{~d} \lambda$; then Proposition 4 gives

$$
\mu(A) \geq \int_{A}|\nabla u|^{2} \mathrm{~d} x+4 \int_{0}^{1} \sqrt{V(s)} \mathrm{d} s \mathcal{H}^{n-1}(S(u) \cap A),
$$

concluding the proof of the liminf inequality.

As for the upper bound, given a function $u \in G S B V(\Omega)$ and $v=1$ let $\left(u_{\varepsilon}, v_{\varepsilon}\right)$ be a recovery sequence for the Ambrosio-Tortorelli approximation with the potential $V$ independent of $\varepsilon ;$ i.e., such that, having we set

$$
G_{\varepsilon}^{0}(u, v)=\int_{\Omega}\left(v^{2}|\nabla u|^{2}+\frac{V(v)}{\varepsilon}+\varepsilon|\nabla v|^{2}\right) \mathrm{d} x,
$$

we have $\lim _{\varepsilon \rightarrow 0} G_{\varepsilon}^{0}\left(u_{\varepsilon}, v_{\varepsilon}\right)=G(u, v)$. Recall that (provided $u$ is first approximated by piecewise-smooth functions in a standard way; see e.g., [14] or [13], Sect. 10.1.1) such $\left(u_{\varepsilon}, v_{\varepsilon}\right)$ can be chosen with $\left|\left\{v_{\varepsilon} \neq 1\right\}\right| \leq C \varepsilon$ (see e.g., [11], Sect. 4.2.3). If we plug such a pair in our $G_{\varepsilon}$ we then obtain

$$
\begin{aligned}
G_{\varepsilon}\left(u_{\varepsilon}, v_{\varepsilon}\right) & =G_{\varepsilon}^{0}\left(u_{\varepsilon}, v_{\varepsilon}\right)+\int_{\Omega} \frac{V_{\varepsilon}\left(v_{\varepsilon}\right)-V\left(v_{\varepsilon}\right)}{\varepsilon} \mathrm{d} x \\
& =G_{\varepsilon}^{0}\left(u_{\varepsilon}, v_{\varepsilon}\right)+\int_{\left\{0<v_{\varepsilon}<1\right\}} \frac{V_{\varepsilon}\left(v_{\varepsilon}\right)-V\left(v_{\varepsilon}\right)}{\varepsilon} \mathrm{d} x \\
& \leq G_{\varepsilon}^{0}\left(u_{\varepsilon}, v_{\varepsilon}\right)+C\left\|V_{\varepsilon}-V\right\|_{\infty}=G_{\varepsilon}^{0}\left(u_{\varepsilon}, v_{\varepsilon}\right)+o(1),
\end{aligned}
$$

which shows that $\left(u_{\varepsilon}, v_{\varepsilon}\right)$ is also a recovery sequence for our $\Gamma$-limit.

\subsection{Other applications}

We quickly mention two other applications in which an energy, similar to our $F(u, E)$, is used. 


\subsubsection{Crystalline film on a substrate}

In $[7,19]$, the following energy is introduced:

$$
\mathcal{E}(u, h)=\int_{\left\{0<x_{n}<h\left(x^{\prime}\right)\right\}} W(\nabla u(x)) \mathrm{d} x+\int_{\omega} \sqrt{1+\left|\nabla h\left(x^{\prime}\right)\right|^{2}} \mathrm{~d} x^{\prime}
$$

where: $\omega=\mathbb{R}^{n-1} / \mathbb{Z}^{n-1}$ is the $(n-1)$-dimensional torus, $h: \Omega \rightarrow[0,+\infty)$ is a smooth function, $W: \mathbb{R}^{n \times n} \rightarrow$ $[0,+\infty)$ is a quasiconvex function with growth $p>1$, and $u$ is a $\mathbb{R}^{n}$-valued displacement. This energy is supposed to be a simplified model for a thin layer of crystal (whose reference configuration is $\Omega_{h}=\left\{x=\left(x^{\prime}, x_{n}\right): x^{\prime} \in\right.$ $\left.\Omega, 0<x_{n}<h\left(x^{\prime}\right)\right\}$, the subgraph of $h$ ), deposited on a crystalline substrate $\left\{x: x_{n} \leq 0\right\}$. Due to the mismatch between both crystalline lattices, the crystal layer is stretched at the interface $\left\{x_{n}=0\right\}$. This is expressed by a Dirichlet condition on the displacement $u: u\left(x^{\prime}, 0\right)=\left(\delta x^{\prime}, 0\right)$ (for instance) on this interface $(\delta \neq 0$ is a parameter related to the mismatch). Moreover, it is assumed for simplicity that $\omega \ni x^{\prime} \mapsto u\left(x^{\prime}, x_{n}\right)-\left(\delta x^{\prime}, 0\right)$ is periodic (for all $x_{n}$ ), in other words: $u-\left(\delta x^{\prime}, 0\right) \in W^{1, p}\left(\Omega_{h} ; \mathbb{R}^{n}\right)$.

The effect of the surface tension of the crystal is represented by the term $\int_{\omega} \sqrt{1+|\nabla h|^{2}}$ which penalizes its total surface. A competition occurs between the elastic term of the energy (which would like to release the stress induced by the mismatch: one easily sees that infinitely many vertical fractures can totally release this stress and make the elastic energy as small as wanted) and the surface tension (which would like, on the contrary, the surface of the crystal to be as flat as possible).

In [7], the two-dimensional case $(n=2)$ is considered. The material is supposed to be linear-elastic, that is, $W(\nabla u)=(\mathbf{A} e(u)): e(u)$ where $e(u)$ is the symmetrized gradient of $u$ and the tensor $\mathbf{A}$ defines the Hooke's law of the material. A relaxation formula is given, that extends (17) to any lower-semicontinuous $h$, and any $u \in S B V\left(\Omega_{h} ; \mathbb{R}^{2}\right)$. An Ambrosio-Tortorelli type approximation is introduced, and the result of numerical experiments are shown.

In [19], the last two authors have tried to extend the mathematical results of [7] to higher dimension. Similar results are shown but the proofs are significantly more technical.

When computing the lower semicontinuous envelope of (17) (in some reasonable topology), as in the present paper one computes a lower estimate and show that it is also an upper estimate. Up to minor technical details, the lower estimate is easily deduced from the present paper. The main difficulties arise when showing the upper estimate. In (17), the role of the "hole" $E$ is played by the complement of $\Omega_{h},\left\{x: x_{n} \geq h\left(x^{\prime}\right)\right\}$. It is thus constrained to be a supergraph. One therefore needs to show that any "generalized" supergraph (in some sense) is approximated by smoother sets that are still supergraphs, with almost the same surface tension.

\subsubsection{Water waves}

In a forthcoming study, Chambolle, Séré and Zanini [20] introduce the following problem, which models a periodic water wave in presence of gravity and surface tension:

$$
\min _{V} \min _{u, F}\left\{\frac{\mu^{2}}{\left(V-\int_{F}|\nabla u(x)|^{2} \mathrm{~d} x\right)^{+}}+\beta \int_{F} x_{2} \mathrm{~d} x+\mathcal{H}^{1}(\partial F)\right\} .
$$

Here, $F \subset \omega \times(0,+\infty)$, where $\omega=\mathbb{R} / \mathbb{Z}$ is the one-dimensional torus, and $u$ is such that $u\left(x_{1}, x_{2}\right)-x_{1}$ is periodic (that is, in $H^{1}(F)$ ). The constant $V$ is the volume of $F$. In this setting, the set $F$ represent a "column" of water in the ocean (of depth $V$ ), in a frame which is moving at constant speed -1 , which is the speed of the wave. The speed of the water is proportional to $\nabla u$ (in the frame of the wave, hence $\nabla u-(1,0)$ in a fixed frame). The second term in the energy is a gravity term, while the length $\mathcal{H}^{1}(\partial F)$ is the surface energy of the free surface of the water (more precisely, it should be written $\left.\mathcal{H}^{1}(\partial(F \cup(\omega \times(-\infty, 0])))\right)$.

The functional in (18), and in particular its first term, is inspired by a recent work of Buffoni [18]. The special form of this term rules out the trivial solution $u \equiv(x, 0), F=\omega \times(0, V)$ (no wave, still water). Again, the functional in (18) is a variant of the functional $F(u, E)$ which is studied in the present paper and its relaxation can be derived from the result in Section 2. 


\section{REFERENCES}

[1] G. Alberti and A. DeSimone, Wetting of rough surfaces: a homogenization approach. Proc. R. Soc. Lond. Ser. A Math. Phys. Eng. Sci. 461 (2005) 79-97.

[2] L. Ambrosio and A. Braides, Functionals defined on partitions of sets of finite perimeter, I: integral representation and $\Gamma$ convergence. J. Math. Pures. Appl. 69 (1990) 285-305.

[3] L. Ambrosio and A. Braides, Functionals defined on partitions of sets of finite perimeter, II: semicontinuity, relaxation and homogenization. J. Math. Pures. Appl. 69 (1990) 307-333.

[4] L. Ambrosio and V.M. Tortorelli, Approximation of functionals depending on jumps by elliptic functionals via $\Gamma$-convergence. Comm. Pure Appl. Math. 43 (1990) 999-1036.

[5] L. Ambrosio, N. Fusco and D. Pallara, Functions of Bounded Variation and Free Discontinuity Problems. Oxford University Press, Oxford (2000).

[6] G. Bellettini, M. Paolini and S. Venturini, Some results on surface measures in calculus of variations. Ann. Mat. Pura Appl. 170 (1996) 329-357.

[7] E. Bonnetier and A. Chambolle, Computing the equilibrium configuration of epitaxially strained crystalline films. SIAM $J$. Appl. Math. 62 (2002) 1093-1121.

[8] G. Bouchitté and P. Seppecher, Cahn and Hilliard fluid on an oscillating boundary. Motion by mean curvature and related topics (Trento, 1992), de Gruyter, Berlin (1994) 23-42.

[9] G. Bouchitté, A. Braides and G. Buttazzo, Relaxation results for some free discontinuity problems. J. Reine Angew. Math. 458 (1995) 1-18.

[10] B. Bourdin and A. Chambolle, Implementation of an adaptive finite-element approximation of the Mumford-Shah functional. Numer. Math. 85 (2000) 609-646.

[11] A. Braides, Approximation of Free-Discontinuity Problems. Lect. Notes Math. 1694, Springer, Berlin (1998).

[12] A. Braides, $\Gamma$-convergence for Beginners. Oxford University Press, Oxford (2002).

[13] A. Braides, A handbook of $\Gamma$-convergence, in Handbook of Differential Equations. Stationary Partial Differential Equations, Vol. 3, M. Chipot and P. Quittner Eds., Elsevier (2006).

[14] A. Braides and V. Chiadò Piat, Integral representation results for functionals defined in $S B V\left(\Omega ; \mathbb{R}^{\mathrm{m}}\right)$. J. Math. Pures Appl. $\mathbf{7 5}$ (1996) 595-626.

[15] A. Braides and R. March, Approximation by $\Gamma$-convergence of a curvature-depending functional in Visual Reconstruction. Comm. Pure Appl. Math. $\mathbf{5 8}$ (2006) 71-121.

[16] A. Braides and M. Solci, A remark on the approximation of free-discontinuity problems. Manuscript (2003).

[17] A. Braides, A. Defranceschi and E. Vitali, Homogenization of free discontinuity problems. Arch. Rational Mech. Anal. 135 (1996) 297-356.

[18] B. Buffoni, Existence and conditional energetic stability of capillary-gravity solitary water waves by minimisation. Arch. Ration. Mech. Anal. 173 (2004) 25-68.

[19] A. Chambolle and M. Solci, Interaction of a bulk and a surface energy with a geometrical constraint. SIAM J. Math. Anal. 39 (2007) $77-102$.

[20] A. Chambolle, E. Séré and C.Zanini, Progressive water-waves: a global variational approach. (In preparation).

[21] E. Giusti, Minimal surfaces and functions of bounded variation. Birkhäuser Verlag, Basel (1984).

[22] J.M. Morel and S. Solimini, Variational Methods in Image Segmentation. Progr. Nonlinear Differ. Equ. Appl. 14, Birkhäuser, Basel (1995)

[23] D. Mumford and J. Shah, Optimal approximations by piecewise smooth functions and associated variational problems. Comm. Pure Appl. Math. 42 (1989) 577-685. 